\title{
GENERAL MASSIVE MULTIPLETS IN EXTENDED SUPERSYMMETRY
}

\author{
S. Ferrara, C.A. Savoy and B. Zumino \\ CERN -- Geneva
}

\begin{abstract}
We consider the extended Poincaré superalgebra in four dimensions with general central charge operators. We find the invariances of this algebra and the particle supermultiplets with their internal symmetry properties. Central charges obtained by dimensional reduction appear as a special case in this classification.
\end{abstract}

Ref.TH. 3010-CERN

12 December 1980 
It is well known that in four dimensions the most general supersymmetry algebra, consistent with Poincaré invariance and certain requirements on the $S$ matrix coming from a relativistic quantum field theory, has the following structure $^{l)}$

$$
\begin{aligned}
& \left\{Q_{\alpha}^{i}, Q_{\beta}^{j}\right\}=(\gamma \mu C)_{\alpha \beta} P_{\mu} \delta^{i j}+C_{\alpha \beta} U^{i j}+\left(\gamma_{s} C_{\alpha \beta} V^{i j},\right. \\
& {\left[Q_{\alpha}^{i}, P_{\mu}\right]=0,\left[Q_{\alpha}^{i}, M_{\mu \nu}\right]=i\left(\sigma_{\mu \nu}\right)_{\alpha}^{\beta} Q_{\beta}^{i}} \\
& {\left[U_{j}^{i j} Q_{\alpha}^{l}\right]=\left[V^{i j} Q_{\alpha}^{l}\right]=\left[U^{i j}, U^{l m}\right]=\left[V^{i j} V^{l m}\right]=\left[V^{i} U^{l m}\right]=0}
\end{aligned}
$$

together with additional commutation relations for the Poincaré algebra and the fact that $U^{i j}$ and $V^{i j}$ are Poincaré invariant operators. In Eq. (1), $C$ is the charge conjugation matrix $C=-C^{T}$, and $Q_{\alpha}^{i}$ are Majorana spinors, i.e., $(C \bar{Q})_{\alpha}^{\dot{i}}=Q_{\alpha}^{\dot{I}}$. In the Majorana representation, $Q_{\alpha}^{i}$ are Hermitean operators. The $\mathrm{U}$ and $\mathrm{V}$ are Hermitean operators which belong to the centre of the superalgebra and for that reason are called central charges. From (1) it follows that $U^{i j}=-U^{j i}, V^{i j}=-V^{j i}$. There are no further restrictions on $U$ and $V$ from the Jacobi identities. The central charges satisfy a positivity bound which we will exploit later.

When $\mathrm{U}^{i j}=\mathrm{V}^{i j}=0$, the general classification of the representations of the algebra (1) acting on one-particle physical states has been given ${ }^{2), 3) . ~ T h i s ~}$ was achieved by using the method of induced representations and by noting that the supersymmetry algebra for fixed timelike or lightlike momentum $P^{2} \leq 0$ reduces to a Clifford algebra for $2 \mathrm{~N}$ or $\mathrm{N}\left\langle\mathrm{P}^{2}=0\right)$ creation and destruction operators respectively. It is the aim of the present paper to generalize this classification to the case of the extended supersymmetry algebra (1) with arbitrary central charge operators. In particular we shall classify all possible supermultiplets corresponding to all central charge operators and we shall also exploit the residual symmetries of (1), in the presence of the central charges, which turn out to be subgroups of $U(N)$.

The main motivation of the present analysis is to have a complete classification of massless and massive multiplets and possibly to have a general procedure for a Higgs effect for higher spin states, at least at the level of counting the degrees of freedom. This is in view of the possible relevance of higher spin multiplets of composite fields, which may be used for current particle phenomenology as speculated in the work of Ellis et al. $\left.{ }^{4}, 5\right)$. 
The role of central charges in supersymmetric Lagrangian field theories has been considered by Fayet ${ }^{6)}$ and particular realizations of supermultiplets have also been discussed in Refs 7)-9). Central charges avoid the proliferation of states in massive representations by eliminating a discontinuity in the maximum spin between massive and massless multiplets. For this reason, they play a crucial role in super Yang-Mills theories with a spontaneously broken Yang-Mills group but with an unbroken supersymnetry ${ }^{6)}$. Supermultiplets with central charges also appear in supergravity models, in which case the central charges are gauge symmetries $^{10)}$. Of particular interest are models where mass parameters are introduced via dimensional reduction ${ }^{11}$ ). They provide unique examples of supermultiplets of elementary fields with non-vanishing central charges and spin $J>1$ 12). The role of central charges in connection with non-trivial topological solutions of supersymmetric Yang-Mills theories has also been investigated ${ }^{13}$ ). More recently, supersymmetry algebras with (off-shell) central charges have been used in order to obtain a field representation of certain extended supermultiplets ${ }^{14}$ ) $(N=2, N=4$ Yang-Mills and $N=8$ supergravity) with the correct number of bosonic and fermionic degrees of freedom which ensure the off-shell closure of the supersymmetry algebra.

We consider the anticommutation in (1) in the Weyl basis

$$
\gamma^{\mu}=i\left(\begin{array}{cc}
0 & \sigma^{\mu} \\
-\sigma_{\mu} & 0
\end{array}\right), C=\left(\begin{array}{cc}
\sigma_{2} & 0 \\
0 & -\sigma_{2}
\end{array}\right), \gamma_{5}=\left(\begin{array}{cc}
i & 0 \\
0 & -i
\end{array}\right)
$$

$\gamma_{5}=\gamma^{0} \gamma^{1} \gamma^{2} \gamma^{3}, \sigma_{0}=-\sigma^{0}=I$ and $\sigma_{i}{ }^{\prime} s$ are the Pauli matrices. Correspondingly, we introduce weyl spinors:

$$
\begin{aligned}
Q_{\alpha}^{i}=\left(Q_{L}^{i}\right)_{\alpha} \quad\left(Q_{R}^{i}\right)^{\dot{\alpha}} & =\epsilon^{\dot{\alpha} \dot{\beta}} Q_{\dot{\beta}}^{* i} \\
(\alpha, \beta=1,2 ; \dot{\alpha}, \dot{\beta}=1,2) \quad & =\left(i \sigma_{2} Q_{L}^{* i}\right)^{\dot{\alpha}} \\
\epsilon^{\alpha \beta}=\epsilon^{\dot{\alpha} \dot{\beta}}=\left(i \sigma_{2}\right)_{\alpha \beta}=-\epsilon_{\alpha \beta} & =-\epsilon_{\dot{\alpha} \dot{\beta}}
\end{aligned}
$$

The four-component spinor $Q_{\alpha}(\alpha=1, \ldots, 4)$ has lower and upper components given by $\left(Q_{L}\right)_{\alpha}$ and $\varepsilon^{\dot{\alpha} \dot{\beta}_{Q}{ }_{L \beta}^{*}}$ respectively. In the two-component notation, the anticommutation in (1) becomes 
$-3-$

$$
\begin{aligned}
& \left\{Q_{\alpha}^{i}, Q_{\dot{\beta}}^{* j}\right\}=\left(\sigma_{\mu}\right)_{\alpha \dot{\beta}} P^{\mu} \delta^{i j} \\
& \left\{Q_{\alpha}^{i}, Q_{\beta}^{j}\right\}=\epsilon_{\alpha \beta} Z^{i j} \\
& \left\{Q_{\dot{\alpha}}^{* i}, Q_{\dot{\beta}}\right\}=\epsilon_{\dot{\alpha} \dot{\beta}} Z \\
& Z^{i j}=-V^{i j}+i U^{i j}
\end{aligned}
$$

(4)

For massive states at rest, $\mathrm{p}^{\mu}=(M, \delta)$, we define $2 \mathrm{~N}$ component spinous $\left.{ }^{15}\right)$

$$
Q_{\alpha}^{a}=\left(\begin{array}{l}
Q_{\alpha}^{i} \\
Q^{* \alpha i}
\end{array}\right)
$$

ie.,

$$
\begin{aligned}
Q_{\alpha}^{a} & =Q_{\alpha}^{i}, a=i=1,2, \ldots, N \\
& =Q^{* \alpha i}=\epsilon^{\alpha \beta} Q_{\beta}^{* i}, a=N+i=N+1, \ldots, 2 N
\end{aligned}
$$

$Q_{\alpha}^{a}$ satisfy the reality condition

$$
Q_{\alpha}^{* a}=\epsilon^{\alpha \beta} \Omega_{a b} Q_{\beta}^{b}
$$

with

$$
\Omega^{a b}=-\Omega_{a b}=\left(\begin{array}{cc}
0 & I \\
-I & 0
\end{array}\right)_{a b}
$$

With these notations, (4) and (5) become

$\left\{Q_{\alpha}^{a}, Q_{\beta}^{b}\right\}=\epsilon_{\alpha \beta}\left(\begin{array}{cc}Z & M I \\ -M I & Z^{*}\end{array}\right)_{a b}=\epsilon_{\alpha \beta} \Lambda^{a b}$ 
where the $(2 \mathrm{~N} \times 2 \mathrm{~N})$ matrix $\Lambda^{a b}$ has been written in terms of the $N \times N$ numerical matrices $Z^{i j}$ and $M \delta^{i j}$. The numbers $z^{i j}$ are the eigenvalues of the central charge operators for a given irreducible supermultiplet.

Before considering the supersymetry algebra with central charges, let us recall the classification of massive one-particle states in the absence of central charges. When $z^{i j}=0,(7)$ becomes ${ }^{15}$ )

$$
\left\{Q_{\alpha}^{a}, Q_{\beta}^{b}\right\}=\epsilon_{\alpha \beta} \Omega^{a b} M
$$

These anticommutators define the Clifford algebra with an invariance group SO(4N). In the form (8), the algebra has a manifest $\mathrm{SU}(2) \otimes U \mathrm{Sp}(2 \mathrm{~N}) \subset \mathrm{SO}(4 \mathrm{~N})$ invariance. The spinor charges $Q_{\alpha}^{i}$, which behave like a vector under $S O(4 N)$, remain irreducible with respect to $S U(2) \otimes U S p(2 N): 4 N \rightarrow(2,2 N)$. The fundamental irreducible massive multiplet, i.e., the lowest dimensional supermultiplet, is obtained from the Clifford vacuum $\Omega$, defined by the condition $Q_{\alpha}^{i} \Omega=0(\forall \alpha, i)$, by repeated application of the creation operators $Q_{\alpha}^{* j}$ :

$$
\Omega ; Q_{\alpha}^{* i} \Omega ; Q_{\alpha}^{* i} Q_{\beta}^{* j} \Omega ; \ldots ; Q_{\alpha_{1}}^{* i_{1}} Q_{\alpha_{2}}^{* i_{2}} \ldots Q_{\alpha_{k}}^{* i_{k}} \Omega ; \ldots
$$

These $2^{2 \mathrm{~N}}$ states span the spinorial representation of $S O(4 \mathrm{~N})$ which splits into two irreducible ones, each of dimension $2^{2 \mathrm{~N}-1}$, corresponding to bosons and fermions respectively. The physical states are obtained by decomposing the spinor representation with respect to $S U(2) \otimes U S p(2 N)$ which classify the states of given intrinsic $\mathrm{SU}(2) \mathrm{J}-\mathrm{spin}$, as follows ${ }^{15}$ )

$$
\begin{aligned}
2^{2 N}= & (N+1,1)+(N, 2 N)+\ldots+ \\
& \left(N+1-k,[2 N]_{k}\right)+\ldots+\left(1,[2 N]_{N}\right)
\end{aligned}
$$

The first label is the dimension of the $\mathrm{SU}(2)$ multiplet $(\mathrm{J}=(\mathrm{N}-\mathrm{k}) / 2)$ and $[2 \mathrm{~N}] \mathrm{k}$ denotes the totally antisymmetric traceless representation of USp(2N). All the other massive supermultiplets are obtained by taking a vacuum which is not a singlet under $S U(2) \times U(N)$. One can further decompose the massive supermultiplet given by (9) into "massless" ones. This means considering a suitable subgroup $U(1) \otimes S U(N) \subset S U(2) \otimes U S p(2 N)$ when $U(1)$ is generated by the $S U(2)$ 
$J-$ spin projection, $\lambda$, and the $2 \mathrm{~N}$ representation of $\mathrm{USp}(2 \mathrm{~N})$ decomposes under $U(N)$ as $2 \mathrm{~N} \rightarrow \mathrm{N}+\overrightarrow{\mathrm{N}}$. Then the massive multiplet splits into $2^{\mathrm{N}}$ "massless" multiplets, each of dimension $2^{\mathrm{N}}$, as follows $\left.{ }^{15}\right)$

$$
\begin{aligned}
2^{2 N}= & \left\{\frac{N}{2}, 1\right\}+\left\{\frac{N-1}{2}, N\right\}+\ldots+ \\
& \left\{\frac{N-k}{2},[N]_{k}\right\}+\ldots+\{0,1\}
\end{aligned}
$$

where the curly bracket denotes a "massless" supermultiplet specified by a state of maximal spin projection, $\lambda_{\text {MAX }}$, belonging to the antisymmetric representation $[\mathrm{N}] \mathrm{k}$ of $\mathrm{SU}(\mathrm{N})$, namely

$$
\begin{gathered}
\left\{\lambda_{\operatorname{MAX}},[N]_{k}\right\}=\left(\lambda_{\operatorname{MAX}}[N]_{k}\right)+\left(\lambda_{\operatorname{MAx}}-\frac{1}{2},[N]_{k} \otimes \bar{N}\right)+ \\
\ldots+\left(\lambda_{\operatorname{MAx}}-\frac{l}{2},[N]_{k} \otimes[\bar{N}]_{l}\right)+\ldots+\left(\lambda_{\max } \frac{N}{2},[N]_{k}\right)
\end{gathered}
$$

We now consider the modification of this analysis when the central charges $Z^{i j}$ do not vanish. If we perform a $U(N)$ transformation on the two component (Weyl) charges $Q_{\alpha}^{\dot{j}}, Q_{\alpha}^{i} \rightarrow U^{i j} Q_{\alpha}^{j}=\widetilde{Q}_{\alpha}^{i}, Q_{\alpha}^{* i} \rightarrow U^{* i}{ }_{Q}^{* j}{ }_{\alpha}^{* j}=\widetilde{Q}_{\alpha}^{* i}$, Eq. (4) remains unchanged. In the rest frame it reduces to

$$
\left\{\tilde{Q}_{\alpha}^{i}, \tilde{Q}^{* j}{ }_{\beta}\right\}=\delta_{\alpha \beta} \delta^{i j} M
$$

In Eq. (5) the $z^{i j}$ matrix transforms as

$$
z^{i j} \rightarrow\left(U Z U^{\top}\right)^{i j}=\widetilde{Z}^{i j}
$$

i.e., the central charges transform like the complex antisymmetrix [-N $]_{2}$ representation of $U(N)$. We can now use a well-known theorem ${ }^{16)}$ which states that for any complex antisymetric matrix $Z=-Z^{T}$, one can find a unitary transformation such that $\tilde{Z}$ has normal form, namely for even $N$, 
$-6-$

$$
\widetilde{Z}=i \sigma_{2} \otimes \hat{Z}_{N / 2} ; \hat{Z}_{N / 2}=\operatorname{diag}\left(z_{1}, \ldots, z_{N / 2}\right)
$$

and for odd $N$

$$
\widetilde{Z}=\left(\begin{array}{c|c}
i \sigma_{2} \otimes \hat{Z}_{\frac{N-1}{2}} & 0 \\
\hline 0 & 0
\end{array}\right)
$$

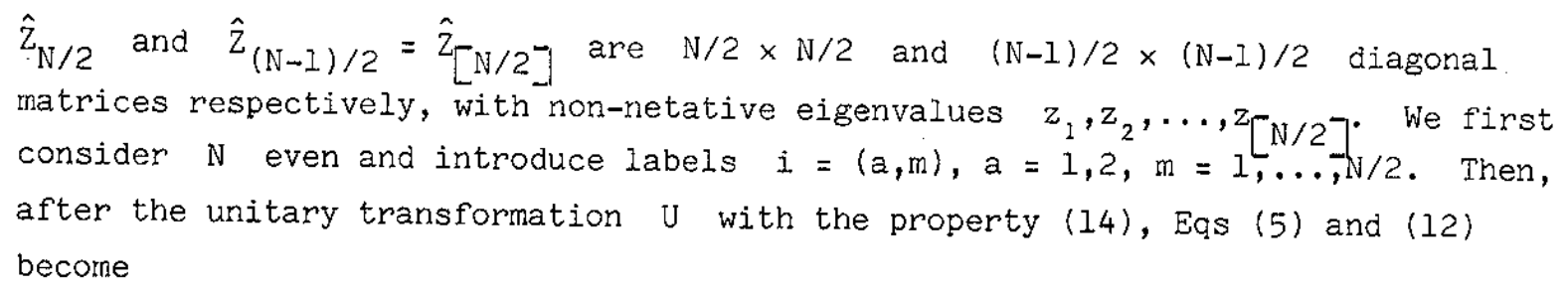

$$
\left\{\widetilde{Q}_{\alpha}^{a m}, \widetilde{Q}_{\beta}^{b n}\right\}=\epsilon_{\alpha \beta} \epsilon^{a b} \delta^{m n} z_{n}
$$

$$
\left\{\tilde{Q}_{\alpha}^{a m}, \tilde{Q}_{b}^{* \beta n}\right\}=\epsilon_{\alpha \beta} \epsilon^{a b} \delta^{m n} M
$$

where

$$
\widetilde{Q}_{a}^{* \alpha m}=\epsilon_{a b} \epsilon^{\alpha \beta} \tilde{Q}_{\beta}^{* b m}
$$

and

$$
\epsilon^{a b}=-\epsilon_{a b}=\left(i \sigma_{2}\right)_{a b}
$$


$-7-$

If we now define the new spinous

$$
S_{\alpha(1)}^{a m}=\frac{\widetilde{Q}_{\alpha}^{a m}+\widetilde{Q}_{a}^{* \alpha m}}{\sqrt{2}} ; S_{\alpha(2)}^{a m}=\frac{\widetilde{Q}_{\alpha}^{a m}-\widetilde{Q}_{a}^{* \alpha m} \cdot(-1)^{a+1}(18)}{\sqrt{2}}
$$

equations (16) and (17) take the form

$$
\begin{aligned}
& \left\{S_{\alpha(1)}^{a m}, S_{\beta(1)}^{b n}\right\}=\epsilon_{a b} \epsilon^{\alpha \beta \delta^{m n}}\left(M+z_{n}\right) \\
& \left\{S_{\alpha(2)}^{a m}, S_{\beta(2)}^{b n}\right\}=\epsilon_{a b} \epsilon^{\alpha \beta} \delta^{m n}\left(M-z_{n}\right) \\
& \left\{S_{\alpha(1)}^{a m}, S_{\beta(2)}^{b n}\right\}=0
\end{aligned}
$$

where the $S_{\alpha(i)}^{a m}$ satisfy the reality conditions

$$
S_{\alpha(i)}^{a m}=\epsilon^{a b} \epsilon_{\alpha \beta} S_{\beta(i)}^{* b m}
$$

and we used the property $\varepsilon^{a c}(-)^{c+l}=\varepsilon^{a c}(-)^{a}$. Because of $(20), a=b=2$ give redundant relations, so we can rewrite (19) only in terms of $s_{\alpha(i)}^{m}=s_{\alpha(i)}^{i m}$ and their complex conjugates:

$$
\begin{aligned}
& \left\{S_{\alpha(i)}^{m}, S_{\beta(j)}^{* n}\right\}=\delta_{\alpha \beta} \delta^{m n} \delta_{i j}\left(M-(-)^{j} z_{n}\right) \\
& \left\{S_{\alpha(i)}^{m}, S_{\beta(j)}^{n}\right\}=\left\{\begin{array}{c}
\left.S_{\alpha(i)}^{* m}, S_{\beta(j)}^{* n}\right\}=0 \\
\quad(\alpha, \beta=1,2 ; i, j=1,2 ; m, n=1, \ldots, N / 2)
\end{array}\right.
\end{aligned}
$$

For $N$ odd we can repeat the same procedure for the first $2(\mathrm{~N}-1)$ spinor charges and the remaining $Q_{\alpha}^{N}$, which corresponds to the zero eigenvalue in (15), satisfies 
$-8-$

$$
\begin{aligned}
& \left\{Q_{\alpha}^{N}, Q_{\beta}^{* N}\right\}=\delta_{\alpha \beta} M ;\left\{Q_{\alpha}^{N}, Q_{\beta}^{N}\right\}=0 \\
& \left\{Q_{\alpha}^{N} S_{\beta(i)}^{* m}\right\}=\left\{Q_{\alpha}^{N}, S_{\beta(i)}^{m}\right\}=0 \quad\left(m=1, \ldots, \frac{N-1}{2}\right)
\end{aligned}
$$

(22)

From (21), one gets the positivity bound

$$
z_{n} \leqslant M, n=1, \ldots, \frac{N}{2}\left(\frac{N-1}{2} \text { for } \operatorname{odd} N\right)
$$

The above inequality also shows that all $z_{n}$ (and therefore $z^{i j}$ ) vanish when $M=0$. We note that the transformations (13) and (18) are the following symplectic rotation on the $Q_{\alpha}^{a}=\left(Q_{\alpha}^{i}, Q^{*} \alpha^{i}\right)$ spinorial charges

$$
\begin{aligned}
& Q_{\alpha}^{a} \rightarrow T_{b}^{a} Q_{\alpha}^{b}=S_{\alpha}^{a} \\
& T=\left[\left(\begin{array}{cc}
A & B \\
-B & A
\end{array}\right) \otimes I_{N / 2}\right] \cdot\left(\begin{array}{cc}
U & 0 \\
0 & U^{*}
\end{array}\right)
\end{aligned}
$$

with

$$
A=\frac{1}{\sqrt{2}}\left(\begin{array}{ll}
1 & 0 \\
1 & 0
\end{array}\right), \quad B=\frac{1}{\sqrt{2}}\left(\begin{array}{cc}
0 & -1 \\
0 & 1
\end{array}\right)
$$

Under the symplectic transformation (24) the matrix $\Lambda^{\text {ab }}$ given by (7) goes into

$$
\tilde{\Lambda}^{a b}=\left(\begin{array}{cc}
0 & M+\hat{z}_{N / 2}^{\otimes \sigma_{3}} \\
-M-\hat{z}_{N / 2} \otimes \sigma_{3} & 0
\end{array}\right)=\Omega^{a b} \rho_{b}
$$


with

$$
\rho_{2 i-1}=\rho_{N+2 i-1}=M+z_{i} ; \quad \rho_{2 i}=\rho_{N+2 i}=M-z_{i} \quad(i=1, \ldots, N / 2)
$$

For odd $\mathrm{N}$ obvious modifications occur. The matrix $\tilde{\Lambda}^{\mathrm{ab}}$ becomes the symplectic metric after rescaling

$$
S_{\alpha}^{a} \rightarrow\left(\frac{M}{P_{a}}\right)^{1 / 2} S_{\alpha}^{a}
$$

It is clear that the transformation $\mathrm{T}_{\mathrm{b}}^{\mathrm{a}}$ is not unique. In particular, the unitary transfornation $U$ is defined as a transformation of the invariance group of the central charge matrix $Z^{j j}$ which will be discussed later.

We now discuss the structure of the fundamental multiplets which arise from the anticommutation relations given by (2l). If no $z_{i}$ 's saturate the positivity bound (23), after the rescaling

$$
S_{\alpha(i)}^{m} \rightarrow\left(\frac{M}{M-(-)^{i} z_{m}}\right)^{1 / 2} S_{\alpha(i)}^{m}
$$

one again obtains the Clifford algebra (8). with

$$
Q_{\alpha}^{a}=\left(\begin{array}{c}
S_{\alpha(i)}^{m} \\
S_{(i)}^{* \alpha m}
\end{array}\right)
$$

For odd $N$ one adds $Q_{\alpha}^{N}$ to the $S_{\alpha(i)}^{m}$ in (25). The Clifford vacuum $\Omega$ defined by the condition $s_{\alpha(i)}^{m}{ }^{\alpha}=0$ has to be doubled, since under a PCT transformation $Q_{\alpha}^{j} \rightarrow i Q_{\alpha}^{* j}$, the central charges transform as follows

$$
z^{i j} \rightarrow-z^{* i j} \quad \text {, i.e., } z_{n} \rightarrow-z_{n}
$$

This has the consequence that the massive multiplet with central charges has twice the (real) degrees of freedom of the spinorial representation associated with the Clifford algebra (8). The invariance group of (8) is $\mathrm{SO}(4 \mathrm{~N})$ and the $2^{2 \mathrm{~N}}$ complex states are classified by $\mathrm{SU}(2) \otimes U \mathrm{USp}(2 \mathrm{~N})$. If $z_{1}=z_{2}=\cdots=z_{q}=$ $=M$, then the $2 q$ operators $S_{\alpha(2)}^{m}$ will vanish and the remaining clifford aigebra will have an invariance $S O(4 \mathrm{~N}-4 \mathrm{q})$ with the states classified by $\mathrm{SU}(2) \otimes$ $\otimes U S p(2 N-2 q)$. The one particle states are then those of a $(N-q)$ extended supersymmetry without central charges, repeated twice. The SU(2) spin will run, inside fundamental multiplet from $J=0$ up to $J=(N-q) / 2$. If all $z_{i}$ saturate 


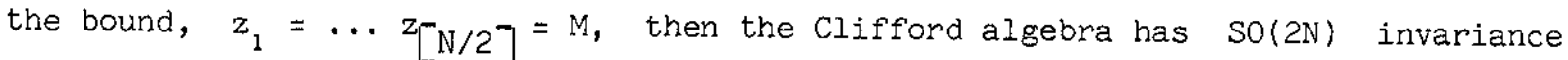
for even $N$ and $S O(2 N+2)$ invariance for odd $N$, the states being classified by $S U(2) \otimes U S p(N)$ and $S U(2) \otimes U S p(N+1)$ respectively. Notice that for even $N$, SO(2N) has the same Clifford algebra of the massless case, $M=0$, in $N$ extended supersymmetry. In the latter case the massless states are classified by $U(1) \times S U(N) \subset S O(2 N)$. Then, by decomposing these $S U(N)$ representations according to $U S p(N) \subset S U(N)$ one can read out how massless multiplets may be recombined into a massive one with central charges, i.e., how the Higgs effect may operate for extended supermultiplets without increasing their maximum $\operatorname{spin}^{6}, 11$ ). We now classify the invariances of the relativistic supersymmetry algebra (1) when $z^{i j} \neq 0$. In a given irreducible supermultiplet, the effect of the $z^{i j} s_{s}$ is to reduce the $U(N)$ invariance of this algebra for $z^{i j}=0$ to the subgroups of $U(N)$ which leave the complex skew symnetric numerical $N \times N$ matrix $Z^{i j}$ invariant. One, therefore, has to classify the orbits of the twofold antisymmetric representations $\left[\mathrm{N}_{-2} \text { of } \mathrm{U}(\mathrm{N}) \text {. The generic orbit has little group (USp(2)) }\right)^{\mathrm{N} / 2}$ for even $N$ or $(\mathrm{USp}(2))^{(\mathrm{N}-\mathrm{l}) / 2} \otimes U(1)$ for odd $\mathrm{N}$. This can be seen, e.g., for even $\mathrm{N}$ by decomposing $\left[\mathrm{N}_{-}^{-}\right]_{2}+\left[\overline{\mathrm{N}}_{-2}^{-} \text {with respect to the subgroup (USp (2)) }\right)^{\mathrm{N} / 2}$

$$
[N]_{2}+[\bar{N}]_{2}=N(1, \ldots, 1)+\operatorname{perm}(2,2,1, \ldots 1) \text {, }
$$

where perm $(2,2,1, \ldots, 1)$ denotes the sum of all the $N / 2((N / 2)-1)$ permutations of the USp(2) subgroups in (USp(2) $)^{N / 2}$. From (27) one must subtract the representation content of the coset space

$$
\begin{aligned}
U(N) /\left[S_{p}(2)\right]^{N / 2} & =\operatorname{Adj} U(N)-\operatorname{Adj}\left[U S_{p}(2)\right]^{N / 2} \\
& =\frac{N}{2}(1, \ldots, 1)+\operatorname{perm}(2,2,1, \ldots, 1)
\end{aligned}
$$

obtaining

$$
[N]_{2}+[\bar{N}]_{2}-\left[\operatorname{Adj} U(N)-\operatorname{Adj}\left(U S_{p}(2)\right)^{N / 2}\right]=\frac{N}{2}(1, \ldots, 1)(29)
$$

An analogous analysis can be done for odd N. We conclude that, in general, the supersymmetry algebra (1) is invariant under (USp(2) $)^{\mathrm{N} / 2}$ (N even) or $(\mathrm{USp}(2))^{(\mathrm{N}-\mathrm{l}) / 2} \otimes \mathrm{U}(\mathrm{l}) \quad(\mathrm{N}$ odd $)$. These are indeed the symmetries of $\tilde{\mathrm{z}}^{\text {ij }}$ given by (14) and (15) in the general case $z_{1}>z_{2} \ldots>z_{[N / 2]}$ i.e., N/2 real parameters. 
The critical orbits have little groups

$$
U S p(2 q) \circledast U(N-2 q) \quad(q=1 \ldots[N / 2])
$$

corresponding to the invariances of $\tilde{z}^{i j}$ in the special one paraneter cases $z_{1}=z_{2}=\ldots=z_{q}>0, z_{q+1}=\ldots=z_{[N / 2]}=0$. This can be checked again by decomposing $[\mathrm{N}]_{2}+[\overline{\mathrm{N}}]_{2}$ with respect to the little groups:

$$
\begin{aligned}
{[N]_{2}+[\bar{N}]_{2} } & =\left(1,[N-2 q]_{2}\right)+\left([q]_{2}, 1\right) \\
& +(1,1)+(29, N-2 q)+\text { h.c. }
\end{aligned}
$$

The coset space decompositions are as follows

$$
\begin{gathered}
U(N) / U S p(2 q) \otimes U(N-2 q)=\operatorname{Adj} U(N)-\operatorname{Adj} U S p(2 q) \\
-\operatorname{Adj} U(N-2 q)=\left([2 q]_{2}, 1\right)+(1,1)+(2 q, N-2 q)+\left(2 q, \frac{(31)}{N-2 q)}\right.
\end{gathered}
$$

Comparing (31) and (30) one sees that

$$
[N]_{2}+[\bar{N}]_{2}-U(N) / U S p(2 q) \otimes U(N-2 q)
$$

contains only one singlet of the corresponding little groups, i.e., the orbits are critical. In general, all the orbits of the $\left[\mathrm{N}^{-}\right]_{2}$ representation of $U(N)$ have little groups

$$
G=U S_{p}\left(2 q_{1}\right) \otimes \ldots U S p\left(2 q_{n}\right) \otimes U(N-2 q)\left(q=\sum_{i} q_{i}\right)^{(32)}
$$

if the eigenvalues of the corresponding matrix $\hat{Z}_{[\mathrm{N} / 2]}$ in (14) or (15) are such that $z_{1}=z_{2}=\ldots=z_{q_{1}}>z_{q_{1}}, \ldots, z_{q_{1}}+q_{2}>\ldots>z_{q}-q_{n}=\ldots=z_{q}>0$, $\left.z_{q+1}=\cdots=z_{[N / 2}\right]_{1}=0$. The matrix $\tilde{Z}^{j j}$ depends in these cases on $n$ parameters. It is evident that (32) classifies all possible non-trivial internal symmetries of the relativistic extended supersymmetry algebra given by (1). Observe that these symmetries of the algebra are also symmetries of the Lagrangian but only in some cases. In the above, we have always considered a single irreducible supermultiplet. If, in a theory, there is more than one, it will not be possible in general to simultaneously bring to normal form the central charge matrices of each supermultiplet. This means that the symmetries $G$ are the largest possible invariances in a given theory. 
In view of this analysis, we expect that in supergravity theories, if supermultiplets with central charges emerge, the possible gauge symmetry $U(N)$ will be broken and the unbroken gauge symmetry will be at most one of the subgroups $G$ given by (32). This result could be relevant in the application of supergravity models to particle phenomenology and in particular in the mass generation for unwanted states in superunification schemes 4 ),5).

Finally, we give the relation between the algebra with central charges obtained by dimensional reduction and the general classification of this paper. We consider a spacetime dimension $D>4$ when a Majorana representation exists ${ }^{17), 18)}$, i.e., $D=10,11,12$ mod.8. The anticonmutators of the $N=1$ supersymmetry algebra in $\mathrm{D}$ dimension are

$$
\begin{aligned}
& \left\{Q_{\alpha}, Q_{\beta}\right\}=-\left(\gamma^{\mu} C\right)_{\alpha \beta} P_{\mu}-\left(\gamma^{A} C\right)_{\alpha \beta} P_{A} \\
& \left(\alpha, \beta=1, \ldots, 2^{[D / 2]} ; \mu=0, \ldots, 3 ; A=4, \ldots, D-1\right) \\
& \gamma_{0}^{2}=-I, \gamma_{i}^{2}=\gamma_{A}^{2}=+I
\end{aligned}
$$

$C$ is the charge conjugation matrix in $D$ dimension. We can always choose a basis in $D$ dimension such that

$$
\begin{aligned}
& P_{\mu}\left(\gamma^{\mu} C\right)_{\alpha \beta, i j}=P_{\mu}\left(\gamma^{\mu} C_{4}\right)_{\alpha \beta} \delta_{i j} \\
& P_{A}\left(\gamma^{A} C\right)_{\alpha \beta, i j}=\left(C_{4}\right)_{\alpha \beta} U_{i j}+\left(\gamma_{5} C_{4}\right)_{\alpha \beta} V_{i j} \\
& \left(\alpha, \beta=1, \ldots, 4 ; i, j=1, \ldots, N=2^{[D / 2]-2}\right)
\end{aligned}
$$

$\left(N=2^{(D / 2-3)} \text { for } D=10 \text { mod.8 when the weyl condition can also be imposed. }\right)^{18)}$ In Eq. (34), $C_{4}$ is the charge conjugation matrix in four dimension and $\mathrm{U}^{\mathrm{ij}}$, $\mathrm{v}^{i j}$ are antisymmetric commuting matrices. In the Weyl basis in four dimension one has

$$
\left(\gamma_{A} C\right)_{\alpha \beta i j} P^{A}=\left(\begin{array}{cc}
\epsilon_{\alpha \beta} Z^{i j} & 0 \\
0 & \epsilon_{\alpha \dot{\beta}} Z^{* i j}
\end{array}\right)
$$


Since

$$
\left(P^{A} \gamma_{A} C\right)\left(P A \gamma_{A} C\right)^{+}=P^{A} P_{A} I
$$

the corresponding $\tilde{Z}^{i j}$ defined by (13) has the form

$$
\tilde{z}=i \sigma_{2} \otimes \hat{z}_{N / 2} \text {, with } \hat{z}_{N / 2}=\sqrt{P_{A} P^{A}} I_{N / 2}
$$

This corresponds to the special critical orbit with an USp(N) invariance. If the theory was massless in $\mathrm{D}$ dimension then

$$
\sqrt{P_{A} P^{A}}=\sqrt{-P_{\mu} P^{\mu}}=M
$$

and the positivity bounds (23) are saturated. In this case the massive four dimensional multiplets are classified by $\mathrm{SU}(2) \otimes U \mathrm{UP}(\mathrm{N})$ and the lowest dimensional multiplet has maximum spin $\mathrm{J}=\mathrm{N} / 4$. 


\section{REFERENCES}

1) R. Haag, J.T. Lopuszanski and M. Sohnius, Nucl. Phys. B88 (1975) 257.

2) A. Salam and J. Strathdee, Nucl. Phys. B76 (1974) 477; Nucl. Phys. B84 (1975) 127.

3) M. Gell-Mann, talk given at the 1977 Washington meeting of the American Physical Society.

4) J. Ellis, M.K. Gaillard, L. Maiani and B. Zumino, Proceedings of the Europhysics Study Conference, "Unification of the Fundamental Particle Interactions", Eds S. Ferrara, J. Ellis and P. van Nieuwenhuizen, Plenum Press (1980).

5) J. Ellis, M.K. Gaillard and B. Zumino, Phys. Lett. 94B (1980) 343;

B. Zumino, Fermilab preprint, FERMILAB-Conf-80/74-THY, talk presented at the XX Int. Conf. on High Energy Physics, Madison (U.S.A.) July (1980);

B. Zumino, CERN preprint TH.2954, talk presented at the Nuffield Supergravity Workshop, Cambridge, June 1980.

6) P. Fayet, ENS-Paris preprint LPTENS $80 / 7$ (1980), Lecture given at the XVII Winter School of Theoretical Physics, Karpacz (Poland), February 1980; P. Fayet, Nucl. Phys. Bl49 (1979) 137.

7) P.H. Dondi and M. Sohnius, Nucl. Phys. B81 (1974) 317; M. Sohnius, Nucl. Phys. B138 (1978) 109.

8) J.T. Lopuszanski, Univ. of Wroclaw preprint No 308 (1974); J.T. Lopuszanski and M. Wolf, Ibid., No 482 (1979).

9) D.Z. Freedman, in Recent Developments in Gravitation, Eds M. Lévy and S. Deser (Plenum Publ. Corp. New York 1979), p. 549.

10) S. Ferrara, J. Scherk and B. Zumino, Nucl. Phys. B121 (1977) 393; C.K. Zachos, Phys. Lett. 76B (I978) 329.

11) E. Cremmer, J. Scherk and J.H. Schwarz, Phys. Lett. 84B (1979) 83.

12) S. Ferrara and B. Zumino, Phys. Lett. 86B (1979) 279.

13) E. Witten and D. Olive, Phys. Lett. 78B (1978) 97.

14) M. Sohnius, K.S. Stelle and P.C. West, Phys. Lett. 92B (1980) 123; idem, Nucl. Phys., in press; idem, in Proceedings of the Europhysics Study Conf. on Unification of Fundamental Interactions, Erice, March 1980, Eds S. Ferrara, J. Ellis and P. van Nieuwenhuizen (Plenum Pub1. Corp., New York, 1980) p. 187; idem, ICTP preprint ICTP/79-80/51, to appear in proceedings of the Nuffield Workshop on Supergravity, Cambridge (U.K.) June 1980;

E. Cremmer, S. Ferrara, K.S. Stelle and P.C. West, Phys. Lett. 94B (1980) 349.

15) S. Ferrara, CERN preprint TH.2957 (1980), plenary talk at the 9th Int. Conf. on General Relativity and Gravitation, Jena (GDR), JuIy 1980; idem, Lectures delivered at the Int. School of Physics "Enrico Fermi" on the Theory of Fundamental Interactions, Varenna (Italy), July 1980.

16) B. Zumino, J. of Math. Phys. 3 (1962) 1055.

17) W. Nahm, Nucl. Phys. B135 (1978) 149.

18) J. Scherk, in Recent Developments in Gravitation, Eds M. Lévy and S. Deser, (Plenum Publ. Corp., New York, 1979 ) p. 549. 